\title{
SECURITY CHALLENGES IN MOBILE ASSISTED LANGUAGE LEARNING IN THE MILLENNIUM FOR EDUCATION
}

\author{
Hassan Soleimani \\ Payame Noor University, Tehran, Islamic Republic of Iran \\ arshia.soleimani@gmail.com \\ Ali Asghar Rostami Abu Saeedi \\ Payame Noor University, Tehran, Islamic Republic of Iran \\ rostamiabu110@yahoo.com \\ Mahboubeh Rahmanian \\ Payame Noor University, Tehran, Islamic Republic of Iran \\ mahboubeh.rahmanian@gmail.com
}

\begin{abstract}
Distance learning technologies enrich learning opportunities due to many advantages like ubiquity and flexibility. Although the usefulness of such technologies in teaching and learning is clear, their testing part is remained to be discussed due to the security issue. Administrators and teachers need to use more authentic and secure distant testing software in which the scores are guaranteed and the testees keep away from cheating. Static and online authentication systems like "username" and "password" and face detection have empowered educational parties to have more reliable testing outcomes. Mobile devices as the necessity of the new millennium need to use authentication software in their testing. Mobile devices with their multimedia course materials provide learners with many optimistic learning opportunities through collaboration, cooperation, interaction and testing. The unique chances of ubiquity, individualization, informality, and spontaneity make the mobile learning of particular importance not only for digital natives but also for teachers, administrators, developers, instructors, and policy makers. Yielding an economical learning opportunity along with providing authentic contexts for collaborative learning is beneficial for the economy of the country in general and for the meaningful and deep learning of the learners. This paper will discuss how authentication techniques have applied to electronic devices like mobile phones.
\end{abstract}

Keywords: security; technology; mobile assisted language learning; testing.

\section{Introduction}

Distant education as a non-boundary education can give the education system noticeable achievement. Previous researchers (e.g., Hannay \& Newvine, 2006; Johnson, Aragon, Shaik, \& Palma-Rivas, 2000; Leasure, Davis, \& Thievon, 2000; Coates, James, \& Baldwin, 2005) agree that this type of learning can yield the same or better results than traditional education in their tests. Virtual or distant education has recently been appreciated by higher education institutes which have radically made a breakthrough in learning frameworks (Robles \& Braathen, 2002). In this type of learning, due to the equal participation of the students, teachers can know their students more appropriately through multimedia like a discussion board, chat rooms, website links, video and audio clip, powerpoint, and set forth. Among these facilities, electronic testing and grading with teachers' feedback are also noteworthy.

There are some disadvantages in using these advanced tools in distance learning, which have made teachers and students unwilling to use them. For example, some technical problems might occur during the instruction or some students might not like to work with such technologies due to their cultural or educational issues. However, an important drawback in online testing is "cheating". It can be explained that the prepared testing tools in online systems can not satisfy the ordinary qualification of tests. In other words, the examination part of virtual systems can hardly come up with the already accepted rules of traditional tests, achieving security. Meeting this requirement can be clearly defined and observed in traditional evaluations; however, in on-line or virtual testing security or preventing fraud is not an easy task. It needs to be noted that in electronic examinations, the students have access to a wide variety of resources in addition to their already established teaching materials. For example, they can use the internet or can have a more capable peer to take their exams. However, we cannot dismiss the possibilities that technological tests provide for us like: "immediate results to faculty and students" (Lorenzetti, 2006, p. 5), ubiquitous and adaptive testing, and immediate feedback (Sjoer \& Dopper, 2003).

E-learning tools like mobile phones can be exploited in this tradition too. As a matter of fact, mobile devices due to their widespread use in telecommunication have recently integrated into the learning environments. Mobile learning as a form of distant education has many beneficial points like acknowledging 
the learners in the learning process and letting learners have a self-ordained syllabus to study in their own time syllabus and in asynchronous means of communication (Shen, Wang, Gao, Novak, \& Tang, 2009).

The purpose of this article is to consider security issue in electronic instructional devices including mobile phones. In the present article, we will examine mobile learning in general and language learning in particular. Considering the potentiality of mobile assisted language learning, we attempt to review some works in this domain. Since electronic learning through the Internet and mobiles have transformed the previous methods of learning and teaching, more studies need to be conducted to lighten these fields for the teachers, students, and administrators. In addition to the techniques of teaching, mobiles also provide many secure ways to assess one's knowledge. However, due to the security issue, mobile phones are not yet completely reliable tools for achieving a secure stance especially for taking further high stake decisions by the administrators. In the present article, we will explain some of the authentication biometrics and introduce a new software in this field which might bring more reliable and secure test scores into existence. However, it will be explained that such authentication biometrics is not yet flourished in mobiles. The issues discussed here about mobile-assisted language learning (MALL) and security in its tests are for adults not studying in formal and traditional contexts. In other words, their job requirements make them study in informal contexts or in their home. Security in MALL and especially its tests can not only provide the opportunity for the learners to study in their own places but also assure the authorities testing the individuals' knowledge itself without any fraud on their part.

\section{Definition and advantages of MALL}

Mobile assisted language learning is learning a language which is facilitated through mobile devices. In other words, mobiles help learners have multimedia course materials and cooperate and collaborate with their colleagues and teachers to enhance their language knowledge. Having no time and place limitation, individualization, spontaneity, and informality can be regarded as the essential features which make MALL a unique opportunity for learning. In spite of space limitations that require them to spend more time on their learning, this tool can exploit the learners' free time in line with enhancing their language knowledge.

Significant opportunities are developed using mobile phones which can let both practitioners and learners transform the traditional formal classrooms and come up with the new demands which digital natives put on the shoulders of developers and teachers. This transition in learning was firstly raised by mobile games and asynchronous messages; however, they have recently been considered by researchers and practitioners as a way to promote learning in social settings through interaction and collaborative learning and through the diverse contextual environments with which the users can work. This portable technology can enrich both the classroom and outside classroom teaching and learning. In other words, the students are led to use the best learning opportunities by the presence of the teacher or in his or her absence.

Electronic learning through the Internet and wireless technologies like mobiles have changed many traditional contexts like previous face to face communication, information accessing, and so on. Naturally, native digitals and those who adopt these technologies to their daily lives can not put these aside in their education. Meaningful and social or contextual learning in these new phones can be the result of their promoted enrichment features like spatial technologies, search technologies, embedded camera technologies, and social networking technologies. Social learning provided through authentic contexts is of primary importance here since authentic context is the channel through which knowledge can be meaningfully acquired. Martin and Ertzberger (2013) use the term here and now for mobile learning, since they believe that it "gives students the opportunity to be in the context of their learning and have access to information that is related to what they are seeing and experiencing at the moment" (p. 77). They believe that this type of learning has brought about the engagement of the learners in the learning process due to the importance given to authenticity and context. In other words, the prevalent atmosphere of learning through games, social networking, and resultant interaction lead many learners to become more involved in their learning process. These refer to the attention factor which is critical in education (Gagne, 2005). The obtained involvement in mobile learning has the advantage of deep and more engaged learning for the learners and in effect has made MALL a very optimistic opportunity for language learning. MALL with its ubiquitous learning of learning anyhow, anytime, and anywhere and its dependency on authenticity and context can bring contextual learning into the scene.

In addition to the features mentioned, mobile users have the opportunity to design and create materials as well to share it with others and to benefit from its informal learning. Informal learning here refers to the learning in which no purposefulness is assumed and the learners can learn unconsciously. Mobile devices due to their enriched context can create this influential condition for the learners. 
Facilitating interaction between learners and teachers and learners themselves through Voice over Internet (VoIP) applications, conferencing system, Skype, and set forth, mobiles can be a useful device for language learning since language can be learned better socially (Kukulska-Hulme \& Shield, 2008). Furthermore, mobiles with their wireless network connection can provide many language learning opportunities for the learners. For instance, they can help learners to gain mastery over writing, speaking, and grammar. Chatting simultaneously between the language students in written and spoken form can also be highlighted. Audio tasks like recording the students' voice and letting them listen to already recorded voices are among the other activities which make mobiles bring both traditional methods of learning and new ones (i.e., interactive ones). Therefore, providing multimedia language learning opportunities, mobiles can bring about "audio and text-based materials as well as encouraging synchronous interaction in L2" (KukulskaHulme \& Shield, 2008, p. 275). These portable devices not only can help learners in providing many personal language activities in formal contexts like providing quizzes and vocabulary items, but also can yield more independent language learners who can take control of their learning (Kukulska-Hulme \& Shield, 2008).

Many studies in MALL have already taken Short Message Service (SMS) or websites as the instruments by which they can deliver contents to the students; however, they have seldom focused on the collaboration of students with each other (Dias, 2002). In another study by Lan et al. (2007), they had learners help each other in improving their reading and listening language skills. However, no knowledge construction and sharing through interaction were observed. Samuels (2003) in another research prepared some chat sessions in which the students could have some unstructured text chats which could not result in the construction of knowledge. In an interesting study by Petersen \& Divitini (2005), they had a community of learners, some of whom had gone to another country and some were at their home. The first group made some audio and video clips about the culture of the foreign country and sent them to the second group through their mobile phones. However, no oral interaction took place between the learners. Yang and Chen (2012) published a paper in which they investigated the effects of a mobile game on L2 learning. They found that the Android-based mobile vocabulary game could teach the university students some new words. In addition, they found that the students had positive perception about such vocabulary games for enhancing their word repertoire. Wang and Shih (2015) in another study investigated the self-paced use of smartphones on learning EFL vocabularies. Ninety-three Taiwanese students received two methods of teaching English vocabularies for 15 weeks. It was unravelled that the group receiving the mobile vocabulary learning performed better than the control group. In addition, the students indicated positive attitudes towards such mobile learning treatment.

Liu and Chen (2015) in an interesting study examined the ways of learning English phrases. They found out that the group who had the treatment of learning phrases by taking photos through their mobiles performed better than the control group with better perceptions towards such electronic activities. Wang (2016) in another research attempted to examine the effect of Mobile-Assisted Classical Chinese (MACC) system on the learners. They found out that the participants in MACC group system demonstrated a good motivation compared to the control group. In addition, Wang (2016) revealed that since mobile-assisted learning system is more valuable due to the chance of learning anytime and anywhere, they are more willing to result in self-regulated learning. Another point in the article is that they draw our attention to the culture of the students and the available electronic devices in managing such classes.

In an exploratory study, Rodríguez and Cumming (2016) investigated iPad language application on learning some language skills like receptive vocabulary, expressive vocabulary, and sentence formation. The participants who had some language disabilities could perform better in the iPad language building application of Language Builder than the control group in sentence formation but not in expressive and receptive vocabulary field. In other words, they concluded that Language Builder application could enhance the sentence formation skills in learners with language disabilities. Sung, Hwang, and Chang (2013) in an interesting study suggest a problem-posing strategy in which they highlight collaborative mobile learning. Problem-posing-based mobile learning was compared with the conventional mobile learning approach and traditional problem-posing approach. They found out that the problem-posing strategy was very useful for the elementary students in that it could improve their learning, culture identity, and self-efficacy in group achievement.

Demouy et al. (2016) in another study investigated learner initiated practices using mobile technology. In fact, they examined the distant language learning of the students. They used a survey and an interview to understand the effect of mobile learning on adult learners. The learning of the students, as well as their motivation, were seen in five languages at different levels. In conclusion, they found out that the students underscored their high, diverse, and pleasant exposure to the target language in the research. They suggested mobiles to the educators since they can have very promising results for the students. It can be inferred that 
using mobile devices are very useful instruments for enhancing the students' motivation and learning outcome. They can bring a very positive attitude of the learners towards learning another language.

\section{Security in electronic instructional devices including mobile-assisted language learning}

Despite all the advantages which mobiles dedicate us in teaching and learning, one aspect of their testing software needs more attention. Although some good attempts have been made to enhance the security part of testing in computers and laptops and in effect to reduce cheating, as far as we found in the literature, no work has been done to take security part of testing into account. In the following, security in larger systems will be discussed.

Distance learning provided through mobile devices are now increasingly growing in education due to the flexibility they create in time and place and the economic conditions they create for the educational parties. Along with offering fruitful learning opportunities for the learners by policy makers, developers, and administrators, security also needs to be taken into account. An authentication system in electronic learning is very important since it sways such educational courses from unsafety and insecurity. The superficial and primary authentication method is using "username" and "password" which can not be so much relied on due to the many fraud ways which are yet open for the testees. For example, the testee might forget it or otherwise other persons can easily use them and take the test. Safe evaluation requires online authentication software which can reduce the risk fraud as much as possible. Biometrics is another feature which can be resorted to since it uses the behavioural or physiological features in its authentication method (Jain, Flynn, \& Ross, 2008). The combination of this online and password securities can bring about safer environments so far. In fact, the term biometrics generally refers to some features in human beings such as fingerprints, face, hand geometry, voice and iris which can lead to a safer world for the digital instruments. Fingerprints as one of this biometrics have been usually used with a password to secure the identity of the individuals. Typing username and putting one's finger on the scanner have also some disadvantages (Phillips, Martin, Wilson, \& Przybocki, 2000). Firstly, the system might not accept even the true fingerprints and falsely reject them. The second problem might be with the quality of the taken pictures which can not yield the proper identity characteristics of each person. The ridge and valley frequency in fingerprints might be a good resource for detecting different fingers and in effect individuals, however, they can not be dependable.

Similarly, face or voice recognition as biometric technologies are not yet very dependable characteristics since a family member or twins yet might create some problems. Face recognition as one of the biometric techniques needs to distinguish different faces at diverse circumstances. Since they are very accurate in their detections and less extraneous variables can change their results, they can be valuable authentications. "These systems have gained remarkable attention among the researchers because of the real time challenges of face images such as expression variation, pose variation with large rotation angles, partial occlusion with objects, aging and complex lighting" (Rose, Suruliandi, \& Meena, 2015, p. 148).

However, since they work with face descriptors which describe the features of the face (Zhao, Chellappa, Phillips, \& Rosenfeld, 2003), much newer descriptors need to be defined by the system to reduce their weaknesses. In addition, since these systems need delicate recognition of the face characteristics, they can not give true results in different environments. This issue becomes more highlighted when the ubiquitous feature of mobile phones comes into our mind. Furthermore, face recognition software need to extract two types of information (i.e., holistic and local feature) from the face of the individuals, both of which need many detailed calculations (Shan, Gong, \& McOwan, 2009; Tian, 2004). In other words, the holistic appearance recognition features deal with the general image of the face while the local features only concern with some points in the face. Calculating such computations on the pixels of the face needs more accurate new local texture descriptors which itself requires more statistical computations. Nevertheless, "recently, a local texture descriptor namely local directional number pattern (LDN) is introduced to encode the directional information of the structure of a face's texture" (Rose, et al., 2015, p. 147). This new system is believed to be effective in descriptor recognition.

Speaker recognition, on the other hand, is the authentication system by which the identity of the individuals can be revealed through their spoken utterances. For a voice to be recognised, two processes should be undergone: identification and verification (Pal \& Saha, 2015). The first one refers to recognising one's speech and the second one concerns with comparing it with the database in order to accept or refuse the concerned voice. Pal and Saha (2015) believe that through voice conversion (VC) approach, individuals can change their voice and put themselves instead of the real person and in effect fraud the speaker identification (SID) and speaker verification (SV) systems. Todays, voice conversion methodologies have progressed in such a case that individuals can easily change their voice to come up with the desired database of the systems. Therefore, SID and SV systems can be easily attacked by such voice conversions. Therefore, 
we need more delicate SID and SV systems which can detect the natural from the fake voices. Pal and Saha (2015) hold that if the score for VC could be objectively placed at a range, spoofing the system can be hardly achieved.

Fingerprint and iris detection are good features that if the system could take high-quality pictures, good bases can be provided upon which the authorities can take appropriate decisions. Iris biometrics is usually believed to be appropriate when individuals are set in less than one-meter distance from the screen. Although iris has this limitation, it "has the great mathematical advantage that its pattern variability among different persons is enormous" (Daugman, 2004, p. 21). Furthermore, compared to face detection, it yields more fixed database. In other words, among other biometrics, iris is the one which can not change by passing time and is more protected inside the eyelid. In addition, it does not have the usual deficiency of fingerprints in threedimensional structures. It is proposed that natural dilation of the iris due to disease or light can not affect its reliability (De Marsico, Nappi, Riccio, \& Wechsler, 2015). Anyway, although iris is held to be an accurate biometrics, its disadvantage is the distance from which the students have to stand from the system and the period of time the system requires to detect the features in it. In general, biometric technologies are progressing continuously which can contribute the language learning field to improve its usefulness and efficiencies. However, the combination of these technologies is much better than working on one individual biometrics feature, each of which has some problems.

Although the defining components of biometrics (i.e., fingerprints, using voice or facial recognition, or iris detection; García-Hernández \& Paredes, 2005) can not be trustworthy due to their demerits during test administration, facial detection is believed to be a better indicator of the students (Guillén-Gámez, GarcíaMagariño, \& Romero, 2015). In fact, silent face recognition and observing no extra demand on the part of the test takers are determinant factors in proving its superiority. Face recognition as a biometric technique does its work by taking the face of the testees and finding some patterns in it. In other words, these patterns let the developers have a proper picture of the considered testee. Face identification through this type of biometric technology can provide the examination parties with more reliable results. Unlike identification in biometrics, authentication technology deals with the correspondence between the face patterns and the database. Farshchi and Toosizadeh (2011) believe that for a particular face to be recognised, two steps need to be taken into account. Firstly, the face has to be recognised, that is separating it from the background environment and secondly, the features of the face have to be extracted in order to be confident if the face has normal features of the ordinary face of the testee.

Considering these factors, Smowl as an authentication software attempts to bring face authentication into the ground. Ribaric, Fratric and Kis (2005) attempt to explain the premise observed in such software. They hold that this software can prove or obviate the picture it takes from the test takers by a comparison it makes with the first picture it takes at the initial time of testing. In other words, random pictures during tests from the test takers are what it maneuvers on in order to make a comparison with its first picture of the testee in its database. These random pictures can contribute test administrators to prevent test-takers from fraud and in effect collect more reliable scores.

\section{Conclusion}

Nowadays, mobile assisted language learning has a huge capacity which has not yet fully discovered. The facilities it provides in teaching and learning are noteworthy. This study intended to reveal how mobiles can be used to facilitate learning and in particular learning English vocabulary, sentence formation, and set forth. However, the studies we investigated so far have dealt with such issues superficially with the usual pretest and posttest control group design. More sophisticated designs might reveal the nature of these new digital tools better. In addition, students from a wide range of experiences and ages and disabilities need to be taken into account. Such ordinary and handy studies can not propel the research community in the appropriate direction. In general, most of these studies have demonstrated the significantly better performance of the group which uses mobile technology in increasing their vocabulary repertoire or sentence formation. Anyway, this important tool has practical implications for teachers, authorities and students. In other words, implementing mobile technology in teaching and learning contexts are noteworthy since they can create an independent atmosphere for the learners which can provide them with more fruitful learning opportunities like working with different visual and auditory helpers or feedback provider tools. Furthermore, teachers can also use these tools to assess the achievement of the students in the passage of time.

The next point here is these new devices and software on the one hand and the students and teachers on the other hand. In other words, the research in this field needs to take the literacy of the individuals into account since we can not reach the true results if teachers and students can not handle such electronic tools. On the other hand, the basic literacy and experience of the participants are also important in producing 
proper results. Therefore, working on different skills and applications which engage the learners in their learning is recommended for the future studies in this field. The motivation and portability of such devices are very interesting characteristics which make such research useful. More longitudinal and larger and more varied sample sizes can reveal such usefulness in more detail.

However, its testing is yet to be explored due to the essentiality of security. The reliability of the results needs to be assured for taking further high stake decisions by the administrators. The authentication biometrics has not yet fully achieved in mobile devices. Understandably, further research needs to be carried out in this field in order to develop more suitable software for secure testing in MALL. In general, although mobiles are very effective and contribute to electronic learning, their deficiency is in their supporting hardware. As such, by now ordinary devices like mobiles are not good choices for developing such biometrics. Therefore, it can be inferred that at least for the present time we can have biometrics in tests when they are administered in some predetermined offices. In the studies in mobile learning, although they are using to take the advantage of mobility, the studies often treat them as static tools. Future studies need to take this issue into account in order to bring the motto of 'anytime, anywhere' into existence (Levy \& Kennedy, 2005). Therefore, as the regular formal contexts can not come up with this requirement in MALL studies, it might be better to give more value to nontraditional contexts.

\section{Acknowledgments}

This study has been part of a grant-based project by Payame Noor University, Iran.

\section{References:}

Coates, H., James, R., \& Baldwin, G. (2005). A critical examination of the effects of learning management systems on university teaching and learning. Tertiary education and management, 11, 19-36. https://doi.org/10.1007/s11233-004-3567-9

Daugman, J.G. (2004). How iris recognition works, IEEE Trans. Circuits Syst: Video Technol, 14 (1), 21-30. https://doi.org/10.1109/tcsvt.2003.818350

De Marsico, M., Nappi, M., Riccio, D., \& Wechsler, H. (2015). Mobile Iris Challenge Evaluation (MICHE)-I, biometric iris dataset and protocols. Pattern Recognition Letters, 57, 17-23. https://doi.org/10.1016/j.patrec.2015.02.009

Demouy, V., Jones, A., Kan, Q., Kukulska-Hulme, A., \& Eardley, A. (2016). Why and how do distance learners use mobile devices for language learning? The EuroCALL Review, 24(1), 10-24. https://doi.org/10.4995/eurocall.2016.5663

Dias, J. (2002). Cell phones in the classroom: Boon or bane? Calling Japan, 10 (2), 16-21. Retrieved from http://jaltcall.org/cjo/10_2.pdf/

Farshchi, S. M. R., \& Toosizadeh, S. (2014). A safe authentication system for distance education. Computer Applications in Engineering Education, 22(4), 593-603. https://doi.org/10.1002/cae.20583

Gagne, R. M. (2005). Principles of instructional design (5th ed.). Belmont, CA: Thomson/Wadsworth.

Garcia-Hernández, J., \& Paredes, R. (2005). Biometric identification using palmprint local features. Biometrics on the Internet, 11.

Guillén-Gámez, F. D., García-Magariño, I., \& Romero, S. J. (2015). Analysis of the Perception of Students about Biometric Identification. International Journal of Web-Based Learning and Teaching Technologies (IJWLTT), 10(3), 1-18. https://doi.org/10.4018/ijwltt.2015070101

Hannay, M., \& Newvine, T. (2006). Perceptions of distance learning: A comparison of online and traditional learning. Journal of Online Learning and Teaching, 2(1), 1-11. Retrieved from: http://jolt.merlot.org/05011.htm

Jain, A., Flynn, P., \& Ross, A. A. (Eds.). (2008). Handbook of biometrics. Springer. https://doi.org/10.1007/978-0-387-71041-9

Johnson, S. D., Aragon, S. R., Shaik, N., \& Palma-Rivas, N. (2000). Comparative analysis of learner satisfaction and learning outcomes in online and face-to-face learning environments. Journal of interactive learning research, 11(1), 29.

Kukulska-Hulme, A., \& Shield, L. (2008). An overview of mobile assisted language learning: From content delivery to supported collaboration and interaction. ReCALL, 20(03), 271-289. https://doi.org/10.1017/s0958344008000335

Leasure, A. R., Davis, L., \& Thievon, S. L. (2000). Comparison of student outcomes and preferences in a traditional vs. world wide web-based baccalaureate nursing research course. Journal of Nursing Education, 39(4), 149-154.

Levy, M., \& Kennedy, C. (2005). Learning Italian via mobile SMS. Mobile learning: A handbook for educators and trainers, 76-83.

Liu, P. L., \& Chen, C. J. (2015). Learning English through actions: a study of mobile-assisted language learning. Interactive Learning Environments, 23(2), 158-171. https://doi.org/10.1080/10494820.2014.959976

Lorenzetti, J. P. (2006). Proctoring Assessments: Benefits \& Challenges. Distance Education Report, 10(8), 5-6.

Martin, F., \& Ertzberger, J. (2013). Here and now mobile learning: An experimental study on the use of mobile technology. Computers \& Education, 68, 76-85. https://doi.org/10.1016/j.compedu.2013.04.021

Pal, M., \& Saha, G. (2015). On robustness of speech-based biometric systems against voice conversion attack. Applied Soft Computing, 30, 214-228. https://doi.org/10.1016/j.asoc.2015.01.036

Petersen, S., \& Divitini, M. (2005). Language Learning: From Individual Learners to Communities. In: Proceedings of the IEEE International Workshop on Wireless and Mobile Technologies in Education (WMTE), Washington, DC, USA: IEEE Computer Society, 169-173. https://doi.org/10.1109/wmte.2005.41

Phillips, P. J., Martin, A., Wilson, C. L., \& Przybocki, M. (2000). An introduction evaluating biometric systems. Computer, 33(2), 56-63. https://doi.org/10.1109/2.820040

Ribaric, S., Fratric, I., \& Kis, K. (2005, September). A biometric verification system based on the fusion of palmprint and face features. In ISPA 2005. Proceedings of the 4th International Symposium on Image and Signal Processing and Analysis, 2005 (pp. 12-17). https://doi.org/10.1109/ispa.2005.195376

Robles, M., \& Braathen, S. (2002). Online assessment techniques. Delta Pi Epsilon Journal, 44(1), 5-15.

Rodríguez, C. D., \& Cumming, T. M. (2016). Employing Mobile Technology to Improve Language Skills of Young Students with Language-Based Disabilities. Assistive Technology, (just-accepted). https://doi.org/10.1080/10400435.2016.1171810 
Rose, R. R., Suruliandi, A., \& Meena, K. (2015). Local texture description framework-based modified local directional number pattern: a new descriptor for face recognition. International Journal of Biometrics, 7(2), 147-169. https://doi.org/10.1504/ijbm.2015.070928

Samuels, J. (2003). Wireless and handheld devices for language learning. Proceedings of the 19th

Annual Conference on Distance Teaching and Learning, Madison, WI. Retrieved from http://www.uwex.edu/disted/conference/Resource library/proceedings/03 50.pdf

Shan, C., Gong, S., \& McOwan, P.W. (2009). Facial expression recognition based on local binary patterns: A comprehensive study. Image and Vision Computing, 27 (6), 803-816. https://doi.org/10.1016/j.imavis.2008.08.005

Shen, R., Wang, M., Gao, W., Novak, D., \& Tang, L. (2009). Mobile learning in a large blended computer science classroom: System function, pedagogies, and their impact on learning. IEEE Transactions on Education, 52(4), 538-546. https://doi.org/10.1109/te.2008.930794

Sjoer, E., \& Dopper, S. M. (2003, May). Are the promises of online assessment being proved in practice. In A case study into what conditions should be met in order to use online assessment successfully, Sefi Proceedings.

Sung, H. Y., Hwang, G. J., \& Chang, Y. C. (2013). Development of a mobile learning system based on a collaborative problemposing strategy. Interactive Learning Environments, 24(3), 1-16. https://doi.org/10.1080/10494820.2013.867889

Tian, Y.L. (2004). Evaluation of face resolution for expression analysis. In Computer Vision and Pattern Recognition Workshop: CVPRW, 4, 82. https://doi.org/10.1109/cvpr.2004.334

Wang, Y. H. (2016). Could a mobile-assisted learning system support flipped classrooms for classical Chinese learning? Journal of Computer Assisted Learning. https://doi.org/10.1111/jcal.12141

Wang, Y. H., \& Shih, S. K. H. (2015). Mobile-assisted language learning: Effects on EFL vocabulary learning. International Journal of Mobile Communications, 13(4), 358-375. https://doi.org/10.1504/ijmc.2015.070060

Yang, T-Y., \& Chen, H-J. (2012). Investigating the effects of a mobile game on EFL learners' vocabulary learning. In J. Colpaert, A. Aerts, W-C. V. Wu, \& Y-C. J. Chao (Eds.), The medium matters: Proceedings 15th International CALL Conference (pp. 697-700).

Zhao, W., Chellappa, R., Phillips, P.J., \& Rosenfeld, A. (2003). Face recognition: A literature survey. ACM Computing Surveys, 35 (4), 399-458. https://doi.org/10.1145/954339.954342 